\title{
Anti-influenza $\mathrm{H7}$ human antibody targets antigenic site in hemagglutinin head domain interface
}

\author{
Jinhui Dong, ${ }^{1}$ Iuliia Gilchuk,, ${ }^{1}$ Sheng Li, ${ }^{2}$ Ryan Irving, ${ }^{1}$ Matthew T. Goff, ${ }^{1}$ Hannah L. Turner, ${ }^{3}$ Andrew B. Ward, ${ }^{3}$ \\ Robert H. Carnahan, ${ }^{1,4}$ and James E. Crowe Jr. ${ }^{1,4,5}$ \\ 'Vanderbilt Vaccine Center, Vanderbilt Medical Center, Nashville, Tennessee, USA. ${ }^{2}$ Department of Medicine and Biochemical Sciences, School of Medicine, UCSD, San Diego, California, USA. ${ }^{3}$ Department of \\ Integrative Structural and Computational Biology, Scripps Research Institute, La Jolla, California, USA. ${ }^{4}$ Department of Pediatrics and ${ }^{5}$ Department of Pathology, Microbiology and Immunology, Vanderbilt \\ University Medical Center, Nashville, Tennessee, USA.
}

\begin{abstract}
Although broadly protective, stem-targeted Abs against the influenza A virus hemagglutinin (HA) have been well studied, very limited information is available on Abs that broadly recognize the head domain. We determined the crystal structure of the HA protein of the avian H7N9 influenza virus in complex with a pan-H7, non-neutralizing, protective human Ab. The structure revealed a B cell epitope in the HA head domain trimer interface ( $\mathrm{TI})$. This discovery of a second major protective TI epitope supports a model in which uncleaved HA trimers exist on the surface of infected cells in a highly dynamic state that exposes hidden HA head domain features.
\end{abstract}

\section{Introduction}

Avian influenza A virus (IAV) H7N9 strains have caused several outbreaks in humans, with high morbidity and mortality. The potential for H7N9 viruses to acquire the capacity for human-to-human transmission presents an imminent health threat worldwide. In typical infections with IAVs, most Abs recognizing the hemagglutinin (HA) surface molecule are directed to the highly variable but immunodominant HA head domain (1). Although head domainspecific Abs tend to be more restricted in breadth compared with stem $\mathrm{mAbs}$, a few broadly reactive, protective, head domain-targeted $\mathrm{H} 7$-specific mAbs have been described. These include the receptor-binding site-targeted (RBS-targeted) neutralizing $\mathrm{mAb}$ L4A-14 (2), the protomer-protomer HA interface-targeted neutralizing mAb H7.5 (3), and the recently described trimer interfacetargeted (TI-targeted), pan-IAV protective, but non-neutralizing, human Abs (4-7).

Here, we determined the crystal structure of the HA1 subunit of the surface HA protein of the avian H7N9 (SH13) influenza virus in complex with what we believe to be a new type of human $\mathrm{mAb}$, designated $\mathrm{H} 7-200$, that recognizes HA proteins for all representative $\mathrm{H} 7$ viruses tested. The complex structure revealed a B cell epitope in the TI of the head domain of HA that is not surface exposed in the prefusion conformation of the HA trimer. The Ab disrupted HA trimers and protected mice challenged with a lethal dose of $\mathrm{H} 7$ virus.

Authorship note: JD and IG contributed equally to this work.

Conflict of interest: JEC has served as a consultant for Takeda Vaccines, Sanofi

Pasteur, Pfizer, and Novavax; is on the scientific advisory boards of CompuVax and

Meissa Vaccines; and is the founder of IDBiologics Inc.

Copyright: (c) 2020, American Society for Clinical Investigation.

Submitted: December 26, 2019; Accepted: May 21, 2020; Published: August 4, 2020

Reference information: J Clin Invest. 2020;130(9):4734-4739.

https://doi.org/10.1172/JCI136032.

\section{Results and Discussion}

The H7-200 mAb was isolated from a B cell in a sample obtained from an otherwise healthy subject who was naturally infected with isolate A/British Columbia/1/2015 H7N9 (BC15) (8). Analysis of the H7-200 Ab variable gene sequences revealed a low frequency of somatic mutations (Supplemental Table 1; supplemental material available online with this article; https://doi.org/10.1172/ JCI136032DS1). The mAb bound tightly to a recombinant HA, based on the infecting isolate in ELISA (with an $\mathrm{EC}_{50}$ of $6.6 \mathrm{ng} / \mathrm{mL}$ ), and also bound to each of the diverse recombinant $\mathrm{H} 7 \mathrm{HA}$ molecules tested from representative Eurasian H7 viruses: A/Shanghai/02/2013 H7N9 (SH13), A/England/268/1996 H7N7 (EN96), A/Netherlands/219/2003 H7N7 (NL03), Yangtze River Delta lineage A/British Columbia/1/2015 H7N9 (BC15), A/Hunan/02650/2016 H7N9 (HN16), and A/Guangdong/17SF003/2016 H7N9 (GD16); and American H7 viruses: A/Canada/rv504/2004 H7N3 (rCA04) and A/New York/107/2003 H7N2 (NY03) (refs. 9-11 and Figure 1A). The mAb also bound to H15 HA (from the A/wedge-tailed shearwater/Western Australia/2576/1979 H15N9 [wts/WA79] strain) (Figure 1A and Supplemental Table 2), but otherwise did not bind in a heterosubtypic manner when tested with HA proteins from each of the other IAV subtypes (Supplemental Table 3). The H7-200 mAb also bound to a recombinant form of the HA1 subunit (a monomer of the head domain, lacking the HA stem) (Figure 1A and Supplemental Table 2). We compared the binding of H7-200 to that of the previously isolated broad H7-specific mAb H7.5 and the heterosubtypic FluA-20 head domain-specific mAb. In contrast to the FluA$20 \mathrm{mAb}$, the $\mathrm{H} 7-200 \mathrm{mAb}$ reacted more strongly with many $\mathrm{H} 7$ antigens (Ags), including the H7 NYO3 HA that lacks the 220 loop, which is part of the FluA-20 epitope. The H7-200 Ab exhibited heterosubtypic reactivity, in that it bound to the H15 HA, whereas the $\mathrm{H7.5} \mathrm{mAb}$ did not (Figure 1A). When tested in concentrations as high as $10 \mu \mathrm{g} / \mathrm{mL}$, the H7-200 Ab did not possess detectable HAinhibiting activity with the SH13 H7N9 vaccine candidate virus, and 
A

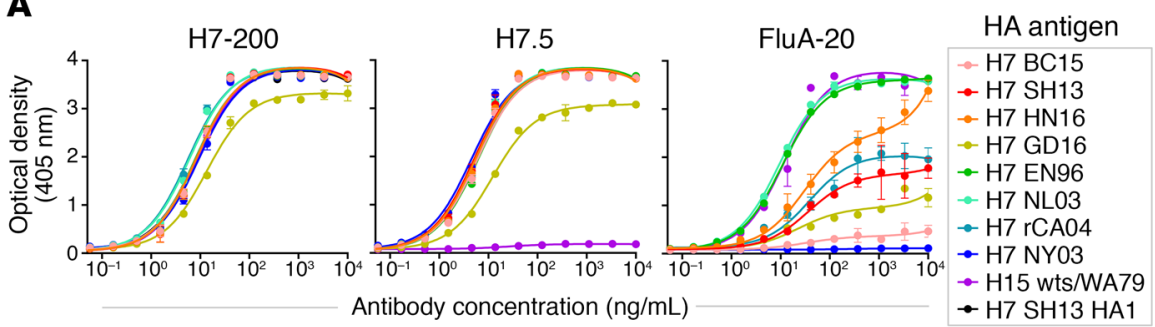

B

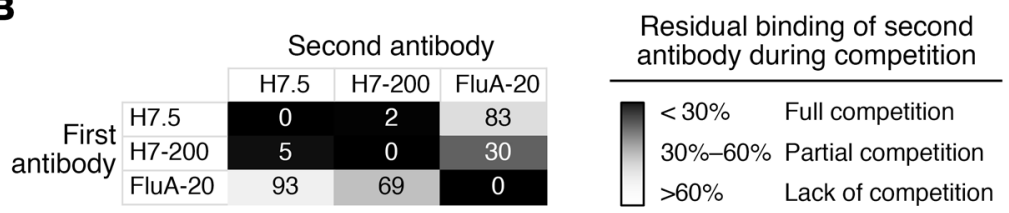

C

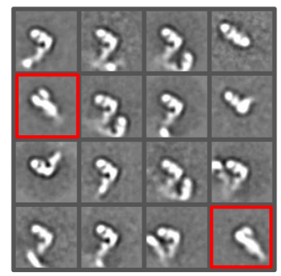

D
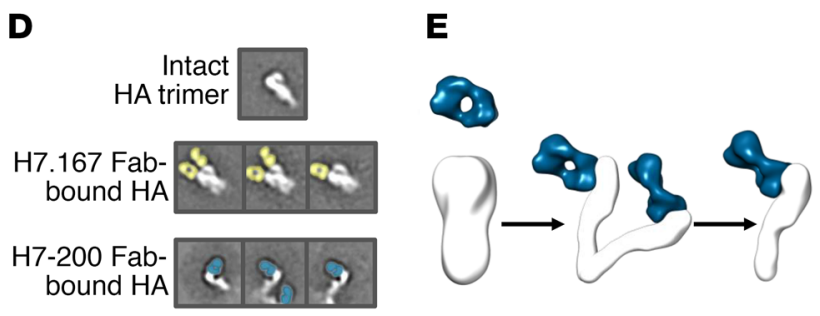

Figure 1. H7-200 is a heterosubtypic HA head domain-specific Ab that disrupts the HA trimer upon binding. (A) $\mathrm{H} 7-200, \mathrm{H} 7.5$, or FluA-20, in serial dilutions, was tested for binding to recombinant soluble proteins from A/Shanghai/02/2013 (H7 SH13), A/British Columbia/1/2015 (H7 BC15), A/Hunan/02650/2016 (H7 HN16), A/Guangdong/17SF003/2016 (H7 CD16), A/England/268/1996 (H7 EN96), A/Netherlands/219/2003 (H7 NL03), A/Canada/rv504/2004 (H7 rCA04), A/New York/107/2003 (H7 NY03), and A/shearwater/Western Australia/2576/1979 (H15 wts/WA79) strains, and detection of binding with a conjugate and colorimetric signal read for optical density at $405 \mathrm{~nm}$ was measured. Data points indicate the mean optical density of 3 replicates, and error bars indicate the SD. (B) Competition-binding assays were performed using biolayer interferometry. A His-tagged H7 SH13 HA1 protein was loaded onto Anti-Penta-HIS biosensors (FortéBio, Sartorius), and the binding of 2 successively applied IgC Abs was tested. (C) Representative 2D class averages of H7-200 bound to $\mathrm{H} 7 \mathrm{SH} 13 \mathrm{HA}$. The majority of the particles were composed of Fab-HA complexes, in which the Fab bound at the trimer interface, thereby disrupting the protomer-protomer interactions and resulting in a splayed open conformation of the HA trimer. We also observed a small minority of intact HA trimers that were not Fab bound (red boxes). (D) Colored class averages showing H7.167 Fab (yellow) or H7-200 Fab (teal) bound to HA protomers (white). The box size for each 2D class average is $295 \times 295 \AA$. (E) Cartoon showing 2 models of the mechanism of Ab-induced trimer dissociation, either principally separation of the head domains or coordinated separation of the full protomer including the head and stem domains.

it exhibited only a minimal level of virus-neutralizing activity in a conventional microneutralization assay with SH13 H7N9 vaccine virus (with an $\mathrm{IC}_{50}$ of $10 \mu \mathrm{g} / \mathrm{mL}$ ). We performed an alternate sensitive neutralization assay using cell impedance measured by realtime cell analysis and did not detect any neutralizing activity for the H7-200 mAb (data not shown).

We used biolayer interferometry to measure whether H7-200 competes for H7 HA1 monomer binding with 2 other known broadly reactive mAbs, H7.5 and FluA-20. MAb H7-200 competed for binding with $\mathrm{H7.5} \mathrm{mAb}$ and partially competed with FluA-20 when H7-200 was added first (Figure 1B).

We next sought to determine the epitope recognized by this pan-H7 mAb. Hydrogen-deuterium exchange mass spectrometry (HDX-MS) studies of the apo H7 HA compared with the complex of H7-200 Fab-H7 HA protein revealed, unexpectedly, that H7-200 bound to the HA trimeric interface including the $\beta$ strand of residues N208 to S216 and part of 220 loop of HA (Supplemental Figure 1). Given the crystal structures of soluble HA trimers, this region of the interface surface was previously thought to be obscured in the trimeric form of HA, until recent studies of trimer interface $m A$ bs elicited by vaccinated individuals (4-7). To our knowledge, the H7-200 mAb is the first documented trimer interface-binding $\mathrm{mAb}$ to be clearly elicited by natural influenza infection. This finding suggests that recognition of the trimer interface is not just an artifact of exposure to unstable HA vaccine Ags, but rather a common feature of responses to infection or vaccination.

We next sought to determine whether mAb H7-200 possesses the antiviral characteristic of TI Abs. A cardinal feature of $\mathrm{TI} \mathrm{Ab}$ function is that $\mathrm{Ab}$ binding to the HA trimer destabilizes the trimeric interface (4, 7). To directly examine the effect of H7-200 Fabs on the HA trimer, we performed negative-stain electron microscopy (nsEM) of HA (uncleaved H7 SH13 [H7 HAO]) in complex with H7-200 Fab (Figure 1C). The native $\mathrm{H} 7 \mathrm{HAO}$ trimer remained in its trimeric conformation during nsEM sample preparation when we studied it alone or in complex with the previously described H7.167 mAb that recognizes the RBS (ref. 12 and Figure 1D). In contrast, we observed that, upon exposure to H7-200 even for 20 seconds (the shortest duration that could be tested), the HAO trimers quickly degraded into Fab-bound monomeric HA, with only a small fraction of Fab-free HA remaining in a trimeric conformation (Figure 1, C-E). These results demonstrate that $\mathrm{H} 7-200$ bound the uncleaved HAO trimer and then dissociated the HA protomers in vitro.

We next tested the effect of prophylactic treatment with H7-200 and found that the mAb conferred a protective effect in vivo. We injected 8-week-old female BALB/c mice $(n=10$ mice per group) via the intraperitoneal route with $200 \mu \mathrm{g}$ mAb 24 hours before intranasal challenge with $10^{4}$ focus-forming units of H7N9 SH13 (IDCDC-RG32A) virus in $50 \mu \mathrm{L}$ PBS. Mice were monitored daily for weight loss, lethality (with $30 \%$ of weight loss considered an endpoint for euthanasia according to IACUC requirements), and clinical score (Figure 2). All mice treated with an unrelated control human mAb (DENV 2D22 directed to dengue virus envelope protein; ref. 13) lost weight, exhibited severe clinical symptoms, and were euthanized. In contrast, all 10 animals treated with H7-200 $\mathrm{mAb}$ had negligible weight changes and low clinical illness scores that were comparable to those seen with a recombinant form of the protective HA stem-specific Ab CR9114 (rCR9114) (14). 

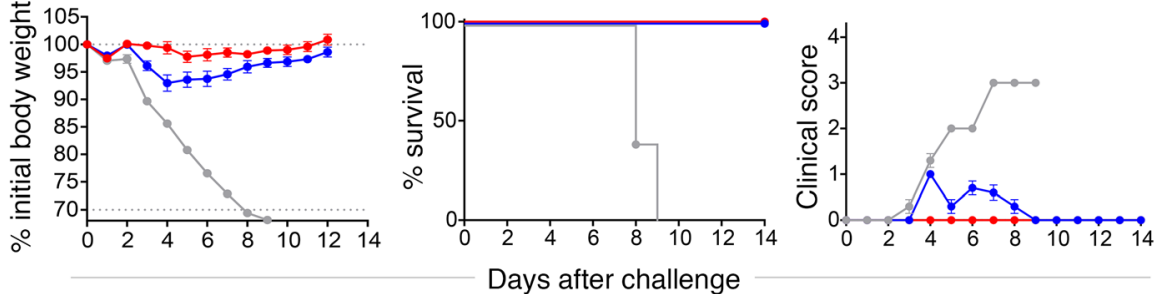

mAb tested: $\rightarrow$ rCR9114 (positive control) $\rightarrow$ H7-200 $\rightarrow$ DENV 2D22 (negative control)

Figure 2. Protective efficacy of H7-200 mAb in mice. Female BALB/c mice weighing 18-20 g were inoculated intraperitoneally with a 200- $\mu$ g dose of H7-200 or a control Ab (DENV $2 D 22$ as a negative control or rCR9114 as a positive control). Twenty-four hours after mAb treatment, anesthetized mice were inoculated intranasally with $10^{4}$ focus-forming units of H7N9 SH13 (IDCDC-RG32A) virus in $50 \mu \mathrm{L}$ sterile PBS. Mice were weighed and monitored daily for BW change and signs of disease for 14 days. Clinical scores were as follows: 1 = slight fur ruffling and/or back arching; 2 = extensive fur ruffling and hunched posture; 3 = minimal mobility, lethargy; 4 = found dead. Mice losing over $30 \%$ of their initial BW were euthanized humanely according to IACUC requirements. Data points indicate the mean values, and error bars indicate the SEM. $n=10$ mice per group.

We determined the crystal structure of H7-200 Fab in complex with H7N9 SH13 HA1 at 3.20-Å resolution (Supplemental Table 4). The Ab-Ag complex was crystallized in spacegroup $\mathrm{P}_{3} 22$, and there were 2 copies of the complex in 1 asymmetric unit (ASU). The 2 structures of the copies were superimposable, with an root mean square deviation (RMSD) of $0.289 \AA$ for the $\mathrm{C} \alpha$ atoms. Because of the high flexibility of the $\mathrm{F}^{\prime}$ fusion domain of HA1, only part of the $\mathrm{F}^{\prime}$ fusion domain had interpretable electron density. The final structure includes the HA1 structure from residue $\mathrm{T} 42$ to residue $\mathrm{V} 310$. Formation of the $\mathrm{Ab}-\mathrm{Ag}$ interface buries a total of $847.8 \AA^{2}$ surface area, to which the heavy chain of H7-200 contributes more than $70 \%$. The $\beta$ strand of residues N208 to S216 of the H7N9 HA1 RBS fit into a shallow groove formed by the three H7-200 heavy-chain complementarity-determining regions (CDRs), heavy-chain strands $C^{\prime}$ and $C^{\prime \prime}$, and the light chain CDR3. The tip of the heavy-chain CDR3 makes additional contacts with adjacent residues on the $\beta$ strand of the HA1 (Figure $3 \mathrm{~A}$ ).

Close examination of $\mathrm{Ab}-\mathrm{Ag}$ interface revealed that the epitope recognized by H7-200 consists of 20 residues, 4 of which are involved in formation of the TI of HA head domains. The paratope on the Fab comprises 24 residues ( 6 from CDRH3, 5 from CDRH1, 6 from CDRH2, $\beta$ strand $C^{\prime}, \beta$ strand $C^{\prime \prime}, 5$ from CDRL3, and 2 from CDRL1) (Figure 3, A-C). Besides 15 polar interactions (salt bridges and hydrogen bonds) between the $\mathrm{Ab}$ and $\mathrm{Ag}$, there are $\pi-\pi$ stacking interactions, e.g., Y92 (H7-200 CDRL3) and Y209 (HA), Y100B (CDRH3) and F213 (HA), and Y100B (CDRH3) and H233 (HA), and hydrophobic interactions between F35/Y52/L97 (H7-200) and L201/V214 (HA) (Figure 3 , B and C). Conceptually, the epitope can be considered to be formed by 2 major regions: (a) a segment of residues N208S216 and L201 (Figure 3B), and (b) the pocket formed by residues K101, V103, I182, H184, D231, and H233, which interacts extensively with CDRH3 Y100B (Figure 3C). As above, H7-200 binds to diverse $\mathrm{H} 7 \mathrm{HA}$ variants and to an $\mathrm{H} 15$ HA from an avian H15N9 virus. To understand the basis for broad recognition of these strains, we aligned HA amino acid sequences from representative $\mathrm{H} 7$ field strains. The important residues in the H7-200 epitope were highly conserved in H7 strains and H15
(Figure 3D and Supplemental Table 5). In contrast, the sequences of HA proteins from other IAV subtypes, including $\mathrm{H} 3$ and H10 HAs, which belong to the same phylogenetic group as H7 HA, were not conserved. These data explain the pan-H7 and $\mathrm{H} 15$ cross-reactive recognition pattern of H7-200 and its lack of other heterosubtypic binding.

The structure revealed that the footprint of the H7-200 mAb identifies what we believe to be a previously unrecognized HA TI epitope that is a new site of vulnerability on the HA trimer. There are 2 separate surfaces involved in the formation of the trimer interface in the head domain. The previously identified TI site recognized by FluA-20 mAbs $(4)$ and others $(6,7,15)$ is located on 1 surface of the interface near the 220 loop (designated here as site TI-1). The H7-200 mAb recognizes the second surface of the interface involving the $\beta$ turn of residues V202-F216 and the $\beta$ strand of residues N240-S246 (designated here as site TI-2). Overlay of the structure of the H7-200-H7.HA1 complex we determined here with that of the complex of the TI-1 mAb FluA-20 with an $\mathrm{H} 3$ head domain onto the prefusion structure of the H7N9 HA trimer (Protein Data Bank [PD]B] ID: 4N5J) clearly showed that H7-200 binds to the TI-2 site, whereas FluA20 binds to the TI- 1 site (Figure 4A). mAb H7.5, which competes with H7-200, recognizes an apical portion of HA head domain near the TI-2 site (Figure 4A).

The TI is not accessible to Ab binding in the "classic" prefusion conformation of the HA trimer. Thus, like TI-1-binding mAbs, $\mathrm{H7}-200$ binding to HA requires large conformational deviation of the HA trimer from the typical closed prefusion conformation. Reversible structural fluctuations or "breathing" motions of HA trimers have been suggested by some previous studies $(3,16)$, but the exact nature of the movements required for recognition of HA by TI Abs is not clear at present. The head domains could transition to an open position with the stem remaining closed, or, alternatively, the entire HA protomers may remain somewhat rigid while separating at both the head and stem in a scissoring action that pivots around a midpoint near the HA1/HA2 border (Figure 4B). The structure of the complex of H7 HA with the H7.5 $\mathrm{mAb}$ may suggest the latter (3). Interestingly, in the structure determined here, we noted some movement within the HA head domain upon binding of H7-200. Superimposition of the vestigial esterase domain of HA1 in the H7-200-H7.HA1 complex with that in the apo H7N9 HA trimer (PDB ID: 4N5J) indicated that the orientations of receptor-binding domains vary significantly relative to the vestigial esterase domain in these crystal structures (Supplemental Figure 2). This finding could be explained by HA1 flexibility resulting in differences in diverse crystals or, alternatively, may suggest that there is a minor hinge function for rigid body rotations located between the receptor-binding domain and the vestigial esterase domain. Such hinge motions could contribute to the conformational fluctuations manifested by crystal structures of TI-binding mAbs in complex with HA1 domains. 
A

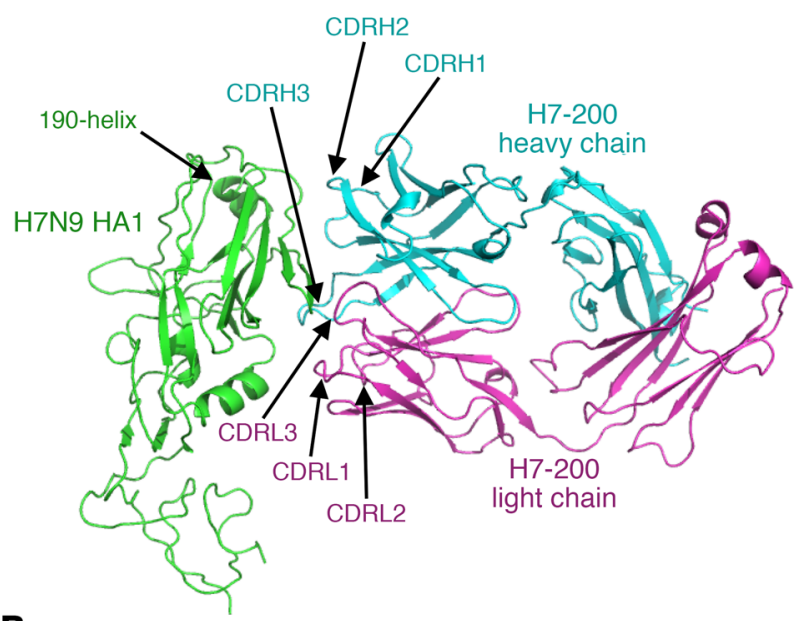

B

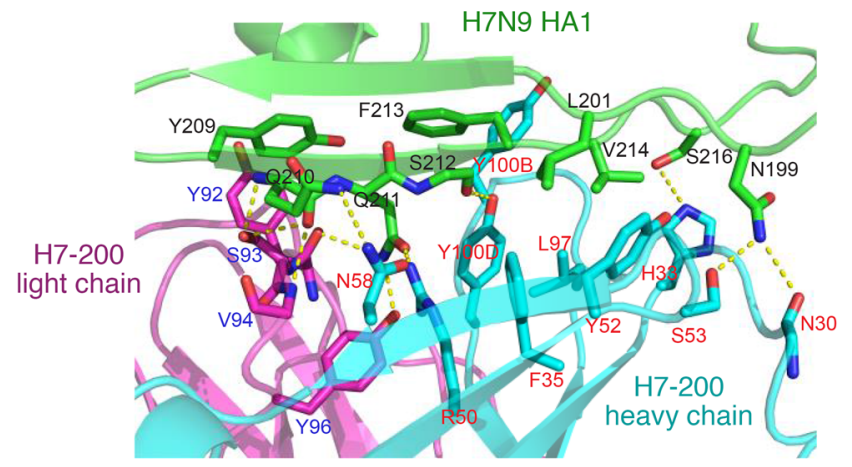

D

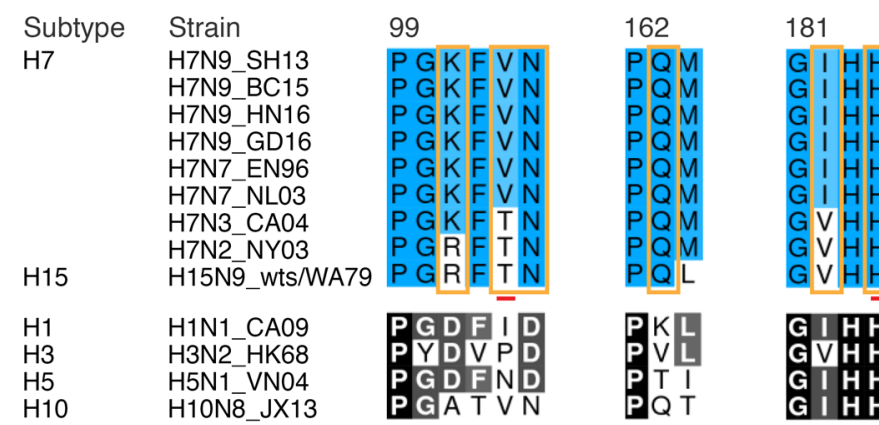

Top view

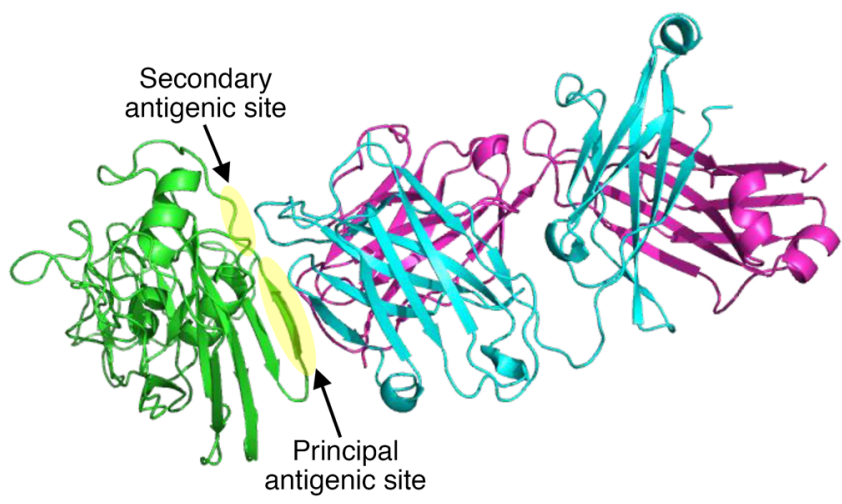

C

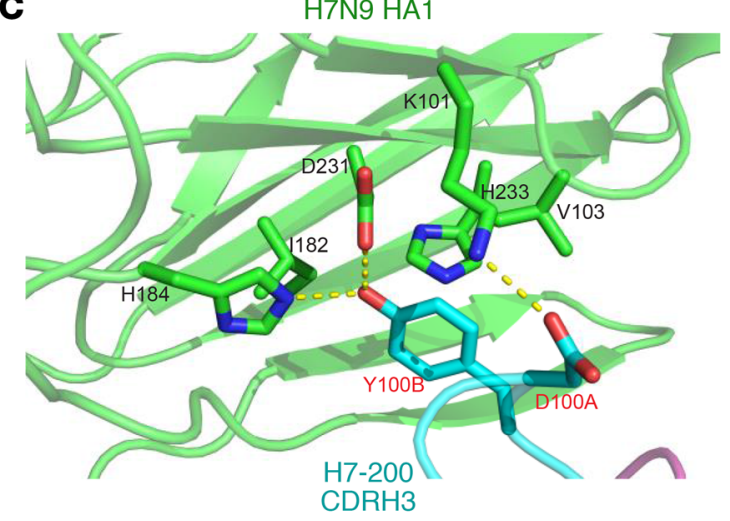

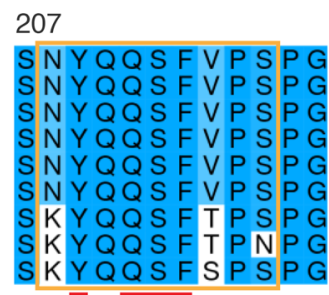

SRY SKKF KPE I A RRSQQT I I PN I G

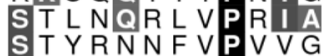

230

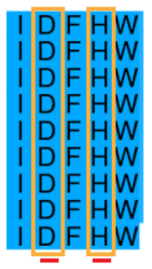

MN Y Y W

I S I YW

ME F FW

Figure 3. Crystal structure and detailed interactions between Fab H7-200 and H7N9 SH13 HA1. All protein components of the Ab-Ag complex are shown in a cartoon representation. The HA1 domain is shown in green, the H7-200 Fab heavy chain in cyan, and the light chain in magenta. (A) Individual CDRs of Fab H7-200, together with the influenza HA1 190 helix of HA1, are labeled. Left and right panels show side and top views of the complex structure. Principal and secondary antigenic sites are indicated with yellow ovals. (B) The principal antigenic site on H7N9 HA recognized by Fab H7-200, including residues Q163, N199-L201, and N208-S216, interacts with the Fab H7-200 heavy-chain strands C' and C', CDRH2, CDRH3, CDRH1, and CDRL3. Residues involved in the Ab-Ag interaction are shown in stick representation. Hydrogen bonds are represented by dashed yellow lines. H7Ng HA residues are labeled in black, residues from Fab H7-200 heavy chain in red, and those from the light chain in blue. Fab H7-200 residues were numbered using the Kabat numbering system. (C) The secondary antigenic site on H7N9 HA recognized by Fab H7-200, consisting of residues K101, V103, I182, H184, D231, and H233, interacts with the tip of the CDRH3 loop of Fab H7-200. (D) Sequence alignment of antigenic sites of several major H7 influenza and H15N9 viruses recognized by Fab H7-200 and H1N1, $\mathrm{H} 3 \mathrm{~N} 2, \mathrm{H} 5 \mathrm{N1}$, and H1ON8 viruses. Antigenic residues are highlighted with brown rectangles, and important residues for Ab-Ag binding in the crystal structure of the Ab-Ag complex are marked with red bars. The first residues of the sequence segments are numbered. Sequence alignment was performed with the multiple sequence alignment software Muscle (17), and the alignment figure was made with the sequence alignment editing software Aline (18).

Thus, mAb H7-200 identifies a second major antigenic site in the HA head domain TI region that is a region of vulnerability for recognition by naturally occurring human Abs with antiviral function. The identification of multiple protective
TI antigenic sites (the TI-1 site previously reported [refs. 4-7] and the TI-2 site reported here) is consistent with a model in which uncleaved HA trimers in the prefusion state transition reversibly into open states that expose the 2 otherwise hid- 
A

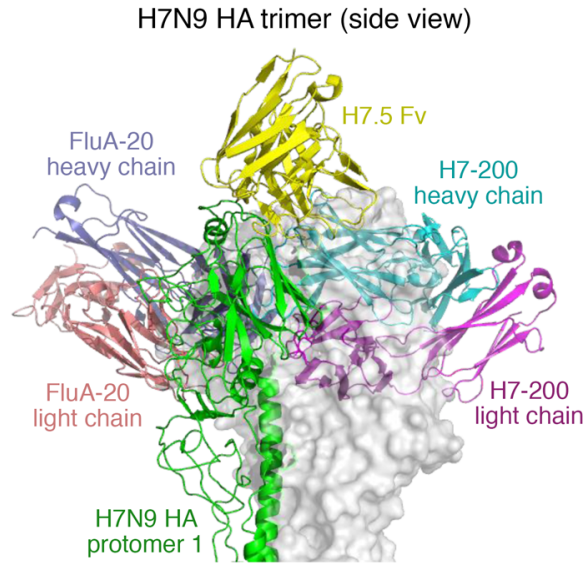

H7N9 HA trimer (top view)

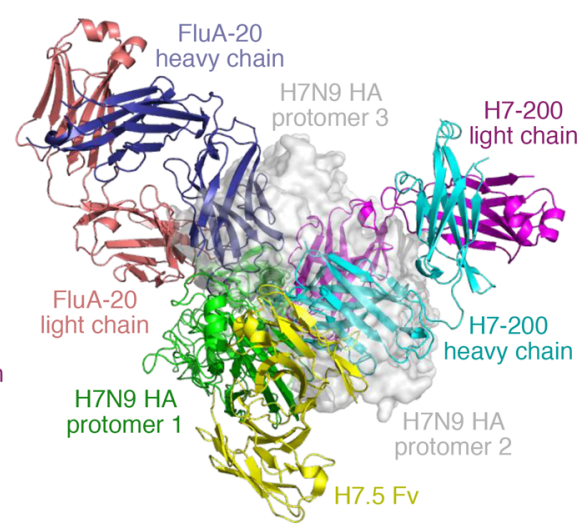

Figure 4. The H7-200 mAb binds a previously unrecognized HA TI epitope. (A) Superimposition of the H7-200 Fab-H7 HA1 complex structure and the $\mathrm{H} 7.5 \mathrm{Fv}-\mathrm{H} 7 \mathrm{HA}$ and FluA-20 Fab-H3 $\mathrm{HA}$ head complex structures onto 1 protomer of the H7N9 HA trimer. Structures of the H3N2 HA head domain H7N9 HA and the H7N9 HA1 domain in the complexes were omitted for clarity. The H7N9 HA protomer 1, onto which the 3 complexes were superimposed, is shown in green, and the other 2 HA protomers are shown as a semitransparent surface representation in gray. (B) Models of conformational changes in the HA trimer.

B

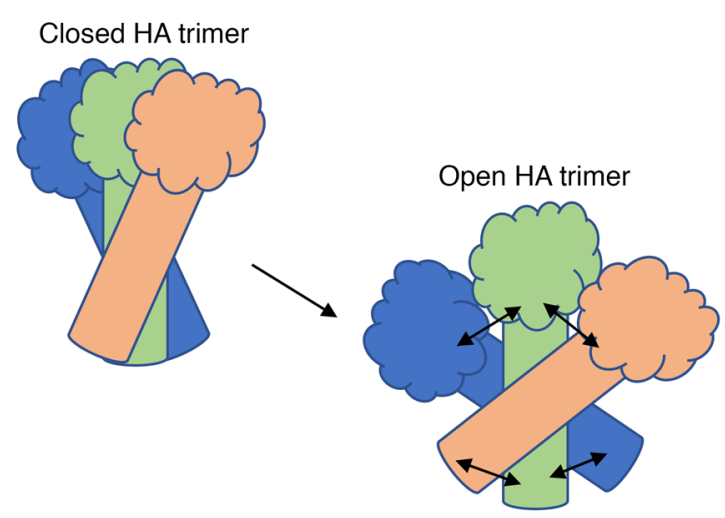

Head-separated model

Full protomer-separated model

den faces of the HA head domain that interact with adjacent protomers. The TI- 1 and TI- 2 antigenic sites represent promising highly conserved, protective B cell epitopes that could be targets for inclusion in the rational design of broadly protective IAV vaccines.

\section{Methods}

Detailed methods are provided in the Supplemental Methods.

Statistics. All statistics were processed using GraphPad Prism software, version 8 (GraphPad Software). For ELISAs, the mean optical density of 3 replicates was determined with SDs. For body weight measurements in mouse experiments, the mean initial body weight percentages were determined and the SEM calculated.

Study approval. The mouse studies were conducted at the Association for Assessment and Accreditation of Laboratory Animal Careaccredited (AAALAC-accredited) laboratory animal research center of Vanderbilt University Medical Center with approval of the IACUC of Vanderbilt University Medical Center.

\section{Author contributions}

JD, IG, SL, RI, HLT, ABW, RHC, and JEC planned experiments. JD, IG, SL, RI, MTG, and HLT performed experiments. JD and JEC wrote the manuscript, and all authors reviewed, edited, and approved the final manuscript. JEC, ABW, and SL obtained funding.

\section{Acknowledgments}

We thank Merissa Mayo for regulatory support and Rachel Nargi and Rachel Sutton of the Vanderbilt University Medical Center for excellent technical support with protein purification. This work was supported by the NIH (U19 AI117905 and contract HHSN272201400024C). The project was supported by Clinical and Translational Science Award (CTSA) UL1 TR002243 from the National Center for Advancing Translational Sciences (NCATS). The contents of this publication are solely the responsibility of the authors and do not necessarily represent the official views of the NCATS, the National Institute of Allergy and Infectious Diseases (NIAID), or the NIH. Data on x-ray diffraction were collected at Beamline 21-ID-G of the Advanced Photon Source, a US Department of Energy (DOE) Office of Science User Facility operated for the Office of Science by Argonne National Laboratory under contract DE-AC02-06CH11357. Use of the Life Sciences Collaborative Access Team (LS-CAT) Sector 21 was supported by the Michigan Economic Development Corporation and the Michigan Technology Tri-Corridor (grant 085P1000817). Support for crystallography was provided by the Vanderbilt Center for Structural Biology.

Address correspondence to: James E. Crowe Jr., Vanderbilt Vaccine Center, 11475 Medical Research Building IV, 2213 Garland Avenue, Nashville, Tennessee 37232-0417, USA. Phone: 615.343.8064; Email: james.crowe@vumc.org. 
1. Angeletti D, Yewdell JW. Is it possible to develop a "universal" influenza virus vaccine? Outflanking antibody immunodominance on the road to universal influenza vaccination. Cold Spring Harb Perspect Biol. 2018;10(7):a028852.

2. Huang KA, et al. Structure-function analysis of neutralizing antibodies to H7N9 influenza from naturally infected humans. Nat Microbiol. 2019;4(2):306-315.

3. Turner HL, et al. Potent anti-influenza H7 human monoclonal antibody induces separation of hemagglutinin receptor-binding head domains. PLOS Biol. 2019;17(2):e3000139.

4. Bangaru S, et al. A site of vulnerability on the influenza virus hemagglutinin head domain trimer interface. Cell. 2019;177(5):1136-1152.e18.

5. Lee J, et al. Molecular-level analysis of the serum antibody repertoire in young adults before and after seasonal influenza vaccination. Nat Med. 2016;22(12):1456-1464.

6. Watanabe A, et al. Antibodies to a conserved influenza head interface epitope protect by an IgG subtype-dependent mechanism. Cell. 2019;177(5):1124-1135.e16.

7. Zost SJ, et al. Canonical features of human antibodies recognizing the influenza hemagglutinin trimer interface. Nat Immunol. In press.

8. Skowronski DM, et al. Avian influenza A(H7N9) virus infection in 2 travelers returning from China to Canada, January 2015. Emerging Infect Dis. 2016;22(1):71-74.

9. WHO. Summary of status of development and availability of $\mathrm{A}(\mathrm{H} 7)$ candidate vaccine viruses. September 2012. https://www.who.int/influenza/vaccines/virus/candidates_reagents/ summary_a_h7_cvv_20120911.pdf?ua=1. Updated September 11, 2012. Accessed June 17, 2020.

10. WHO. Antigenic and genetic characteristics of zoonotic influenza viruses and development of candidate vaccine viruses for pandemic preparedness. March 2017. https://www.who.int/ influenza/vaccines/virus/201703_zoonotic vaccinevirusupdate.pdf?ua=1. Updated March 2, 2017. Accessed June 17, 2020.

11. WHO. Antigenic and genetic characteristics of zoonotic influenza viruses and development of candidate vaccine viruses for pandemic preparedness. February 2019. https://www.who.int/ influenza/vaccines/virus/201902_zoonotic vaccinevirusupdate.pdf?ua $=1$. Updated September 24, 2019. Accessed June 17, 2020.
12. Thornburg NJ, et al. H7N9 influenza virus neutralizing antibodies that possess few somatic mutations. J Clin Invest. 2016;126(4):1482-1494.

13. Fibriansah G, et al. Dengue virus. Cryo-EM structure of an antibody that neutralizes dengue virus type 2 by locking E protein dimers. Science. 2015;349(6243):88-91.

14. Dreyfus C, et al. Highly conserved protective epitopes on influenza B viruses. Science. 2012;337(6100):1343-1348.

15. Bajic $G$, et al. Influenza antigen engineering focuses immune responses to a subdominant but broadly protective viralvepitope. Cell Host Microbe. 2019;25(6):827-835.e6.

16. Das DK, Govindan R, Nikić-Spiegel I, Krammer F, Lemke EA, Munro JB. Direct visualization of the conformational dynamics of single influenza hemagglutinin trimers. Cell. 2018;174(4):926-937.e12.

17. Edgar RC. MUSCLE: multiple sequence alignment with high accuracy and high throughput. Nucleic Acids Res. 2004;32(5):1792-1797.

18. Bond CS, Schüttelkopf AW. ALINE: a WYSIWYG protein-sequence alignment editor for publication-quality alignments. Acta Crystallogr D Biol Crystallogr. 2009;65(Pt 5):510-512. 